\title{
CDKN2A NM_000077.4:C.322G>T
}

National Cancer Institute

\section{Source}

National Cancer Institute. CDKN2A NM 000077.4:C.322 G>T. NCI Thesaurus. Code C146943.

A nucleotide substitution at position 322 of the coding sequence of the CDKN2A gene where guanine has been mutated to thymine. 